\title{
Criminologie
}

\section{Détention des demandeurs d'asile au Canada : des logiques pénales et administratives convergentes}

\section{Delphine Nakache}

Volume 46, numéro 1, printemps 2013

La criminalisation de l'immigration

URI : https://id.erudit.org/iderudit/1015294ar

DOI : https://doi.org/10.7202/1015294ar

Aller au sommaire du numéro

\section{Éditeur(s)}

Les Presses de l’Université de Montréal

ISSN

0316-0041 (imprimé)

1492-1367 (numérique)

Découvrir la revue

Citer cet article

Nakache, D. (2013). Détention des demandeurs d'asile au Canada : des logiques pénales et administratives convergentes. Criminologie, 46(1), 83-105.

https://doi.org/10.7202/1015294ar
Résumé de l'article

Le but de cet article, qui porte sur la détention des demandeurs d'asile au Canada, est de mettre en relief les logiques convergentes entre le traitement pénal et le traitement administratif de l'immigration. Nous démontrons que la " criminalisation " actuelle des demandeurs d'asile facilite la mise en oeuvre de politiques publiques de plus en plus répressives à leur égard, notamment à travers un recours accru à la détention. Nous nous intéressons aussi à la détention des demandeurs d'asile dans les prisons provinciales, alors que le motif de la détention est purement " administratif » (c.-à-d. les demandeurs d'asile ne sont pas des personnes présumées ou reconnues coupables de délits) : ce phénomène n'est pas marginal, puisqu'il concerne un demandeur d'asile sur trois au Canada. Nous montrons alors que l'institution carcérale exerce, au même titre que les " camps d'étrangers ", une fonction de ségrégation spatiale, juridique et sociale ayant pour objectif de marquer les demandeurs d'asile comme une population d'« indésirables ». 


\title{
Détention des demandeurs d'asile au Canada: des logiques pénales et administratives convergentes
}

\author{
Delphine Nakache \\ Professeure adjointe \\ École de développement international et mondialisation (EDIM) \\ Université d'Ottawa \\ dnakache@uottawa.ca
}

\begin{abstract}
RÉSUMÉ - Le but de cet article, qui porte sur la détention des demandeurs d'asile au Canada, est de mettre en relief les logiques convergentes entre le traitement pénal et le traitement administratif de l'immigration. Nous démontrons que la "criminalisation» actuelle des demandeurs d'asile facilite la mise en œuvre de politiques publiques de plus en plus répressives à leur égard, notamment à travers un recours accru à la détention. Nous nous intéressons aussi à la détention des demandeurs d'asile dans les prisons provinciales, alors que le motif de la détention est purement «administratif» (c.-à-d. les demandeurs d'asile ne sont pas des personnes présumées ou reconnues coupables de délits): ce phénomène n'est pas marginal, puisqu'il concerne un demandeur d'asile sur trois au Canada. Nous montrons alors que l'institution carcérale exerce, au même titre que les "camps d'étrangers», une fonction de ségrégation spatiale, juridique et sociale ayant pour objectif de marquer les demandeurs d'asile comme une population d'«indésirables».
\end{abstract}

MotS-CLÉs - Détention, Canada, demandeurs d'asile, droits de la personne, criminalisation de l'immigration.

\section{Introduction}

Les politiques migratoires des pays développés sont aujourd'hui structurées autour de deux axes distincts: l'accueil des migrants «désirables» et le rejet des migrants «indésirables». À l'origine de ce double mouvement, qui n'est pas sans rappeler le système de sélection caractéristique des gated communities (Blandy et al., 2003; Cheng, 2004:1), il y a un processus d'«altérisation», fort bien décrit dans la littérature académique 
(voir sur ce point Derrida, 1968; Bauman, 1991 ; Derrida, 1992; Soguk, 1999; Kapur, 2007; Courau, 2012). En des termes simples, l'altérisation repose sur une différenciation et sur une marginalisation de l'autre visant à mieux faire valoir sa propre identité. Ainsi, par le biais d'une division étanche entre l'identité du «nous» national (présentée comme homogène et définie en fonction d'un héritage distinct) et l'identité d'un groupe «autre» (nécessairement définie en des termes négatifs), les «migrants indésirables» ne sont plus simplement étrangers, mais ils deviennent étranges, donc proprement incompatibles avec les normes et valeurs présumées de la société d'accueil.

C'est à partir de cette perspective que des écrits sur la sécurisation de la migration se sont développés (voir, par exemple, Bigo, 1998a; Bigo, 1998b; Tsoukala et Ceyhan, 2001; Faist, 2002; Crépeau et Nakache, 2006; Huysmans, 2006; Scherrer et al., 2010). Ces travaux analysent de manière critique le lien dorénavant établi entre la sécurité et les questions d'immigration. Ils mettent l'accent sur la mise au ban des étrangers et sur la force des pratiques discursives visant à construire la menace migratoire, en démontrant notamment les processus à travers lesquels les migrants indésirables deviennent responsables des nombreux maux de la société d'accueil (comme le chômage, la crise de l'État-providence, ou encore la perte d'identité nationale). Un des aspects importants du programme de «sécurisation» de la migration est la transformation des logiques de contrôle, avec la coexistence d'un contrôle migratoire à la fois externe (contrôle en amont et aux frontières) et interne (à l'intérieur $\mathrm{du}$ territoire).

Le concept de sécurisation de la migration permet de comprendre l'étendue des politiques migratoires actuelles (ainsi que leurs origines). Toutefois, il ne tient pas suffisamment compte d'un développement récent dans le domaine des migrations: l'interpénétration des logiques administratives et pénales dans le traitement des questions migratoires. Il importe ici de clarifier notre position de départ: la connexion entre contrôle de l'immigration et pénalité n'est pas nouvelle, et les écrits dans ce domaine ont d'ailleurs bien démontré que les politiques migratoires visent, au même titre que les politiques relevant du domaine pénal, à contrôler certaines populations considérées comme «indésirables» du fait des valeurs sociales dominantes (pour une analyse documentaire, voir Fischer et Darley, 2010). Cependant, le contrôle administratif des migrants et le contrôle pénal des délinquants ont régulièrement été abordés comme des «formes relativement cloisonnées de gestion des 
populations» (Fischer et Darley, 2010: 4). Or, on constate que les éléments de ces deux formes de contrôle se conjuguent de plus en plus au sein d'une logique convergente, prenant la forme d'un véritable dispositif de mise à l'écart des migrants indésirables.

Pour illustrer ce point, notre article traite de la détention des demandeurs d'asile (ou revendicateurs du statut de réfugié) au Canada. Il utilise des données de recherche récente dans ce domaine (Nakache, 2011; Cleveland et al., 2012; Global Detention Project, 2012) afin de formuler une réflexion conceptuelle en deux temps. Tout d'abord, nous démontrons la construction du traitement pénal de l'immigration comme problème public au Canada, en analysant la criminalisation actuelle des demandeurs d'asile et la justification du recours accru à leur détention. Ensuite, nous étudions le traitement réservé aux demandeurs d'asile par l'institution carcérale. Au Canada, un demandeur d'asile sur trois est détenu dans une institution de type carcéral alors que le motif de la détention est purement «administratif» (c.-à-d. les demandeurs d'asile sont uniquement détenus à des fins d'immigration, pas pour des raisons criminelles). Il est donc nécessaire d'étudier de plus près ce phénomène, qui n'est pas mineur sur le plan quantitatif. En outre, étant donné le contexte actuel, caractérisé par une volonté marquée du gouvernement canadien de recourir de manière accrue à la détention (et pour plus longtemps), il est important de faire la lumière sur la logique particulière sous-tendant la détention des demandeurs d'asile en milieu carcéral, une logique encore très peu étudiée dans la littérature académique.

Dans une première partie du texte, nous procédons tout d'abord à un survol rapide du cadre juridique actuel propre à l'arrestation et la détention pour des motifs d'immigration au Canada. Nous démontrons par la suite que la volonté du gouvernement canadien de recourir de manière accrue à la détention des demandeurs d'asile constitue une mesure essentiellement punitive à leur égard. Une deuxième partie s'intéresse au sort des demandeurs d'asile au sein de la «chaîne pénale». Nous soulignons dans un premier temps les logiques convergentes entre les «camps d'étrangers» et les prisons. Nous démontrons alors que la prison exerce, au même titre que les «camps d'étrangers», une fonction de mise à l'écart - spatiale et juridique - des demandeurs d'asile. Dans un deuxième temps, nous expliquons que l'enfermement pénal des demandeurs d'asile pour des raisons purement administratives, qui détourne la prison de son sens premier, a aussi pour objectif de les marginaliser davantage. 


\section{La détention: un élément central de la criminalisation des demandeurs d'asile au Canada}

Jusque récemment, la détention en matière d'immigration était conçue comme une mesure au cas par cas au Canada. Les motifs de la détention étaient limités et toute personne détenue avait droit à un contrôle indépendant et régulier des motifs de sa détention. Depuis l'entrée en vigueur fin juin 2012 d'un projet de loi controversé (le projet de loi C-31), la loi actuelle a toutefois été modifiée afin de permettre la détention automatique des demandeurs d'asile faisant partie de la catégorie des «étrangers désignés». Ces derniers sont de plus assujettis à un régime de contrôle des motifs de détention qui diffère de celui visant les autres demandeurs d'asile. Afin de bien comprendre l'ampleur des changements législatifs apportés par le projet de loi C-31, nous expliquons tout d'abord le cadre juridique entourant la détention aux fins d'immigration au Canada, puis nous démontrons par la suite que ces changements récents sont le reflet d'une «criminalisation» des questions migratoires dans le pays.

Le cadre juridique entourant la détention aux fins d'immigration

Depuis l'adoption fin juin 2012 du projet de loi C-31 (Loi visant à protéger le système d'immigration $d u$ Canada $)^{1}$, il existe deux régimes juridiques de la détention aux fins d'immigration: un régime général pour tous les étrangers et un régime distinct pour la catégorie des «étrangers désignés».

Le régime général de la détention

En vertu des dispositions de la Loi sur l'immigration et la protection des réfugiés (LIPR) ${ }^{2}$, les agents de l'Agence des services frontaliers du Canada (ASFC) peuvent arrêter les ressortissants étrangers et les résidents permanents dont ils ont des motifs raisonnables de croire qu'ils sont interdits de territoire et qu'ils constituent un danger pour la sécurité

1. Projet de loi C-31, Première session, quarante et unième législature, 60-61 Elizabeth II, 2011-2012. Le 28 juin 2012, le projet de loi C-31 a reçu la sanction royale. Certaines dispositions du projet de loi C-31 sont déjà en vigueur (comme celles sur la détention), mais la plupart des mesures touchant le système de détermination du statut de réfugié devraient être appliquées au mois de décembre 2012.

2. Loi sur l'immigration et la protection des réfugiés, L.C. 2001, ch. 27. 
publique ou qu'ils se soustrairont vraisemblablement au contrôle, à l'enquête ou au renvoi (art. 55 (1) et (2) de la LIPR) ${ }^{3}$. En outre, les agents de l'ASFC peuvent arrêter et détenir l'étranger dont l'identité n'a pas été prouvée dans le cadre d'une procédure prévue par la présente loi (art. 55 (2) de la LIPR). Finalement, les agents de l'ASFC peuvent détenir le résident permanent ou l'étranger à son entrée au Canada lorsqu'ils l'estiment nécessaire afin que soit complété le contrôle ou lorsqu'ils ont des motifs raisonnables de soupçonner que celui-ci est interdit de territoire pour raison de sécurité ou pour atteinte aux droits de la personne ou internationaux (art. 55 (3) de la LIPR). En somme, il est permis de détenir un étranger en cas de doute sur son identité, lorsque celui-ci risque de ne pas se présenter aux procédures d'immigration, ou lorsqu'il existe un risque pour la sécurité, la criminalité, etc. En réalité, la très vaste majorité des demandeurs d'asile qui sont détenus actuellement le sont soit parce qu'il y a un risque de fuite (l'agent d'immigration estime qu'ils pourraient ne pas se présenter à l'avenir) soit parce qu'il y a un doute concernant leur identité. Un très petit nombre d'entre eux (moins de $6 \%$ ) sont donc détenus au motif qu'ils constituent un risque pour la sécurité ou un danger pour le public (Nakache, 2011 : 47).

La détention dure jusqu'à ce que les autorités de l'immigration aient terminé les contrôles d'identité ou autres vérifications. Durant les 48 premières heures suivant la mise en détention, les agents de l'ASFC doivent examiner les motifs de la détention et peuvent remettre la personne en liberté avec ou sans condition (art. 56 de la LIPR). Après 48 heures de détention, il y a une audience de révision de détention devant la Commission de l'immigration et du statut de réfugié (CISR). Une deuxième audience aura lieu 7 jours plus tard, puis tous les 30 jours (art. 57 de la LIPR). Chaque fois qu'il examine le dossier, le commissaire de la CISR doit décider s'il existe des raisons valables de maintenir la personne en détention. Si ce n'est pas le cas, alors la personne doit être remise en liberté, avec ou sans condition. Le Règlement sur l'immigration et la protection des réfugiés ${ }^{4}$ (RIPR, art. 244 à 250 ) mentionne plusieurs facteurs importants à prendre en compte en vue d'une éventuelle détention et remise en liberté (voir sur ce point Nakache, 2011: 28).

3. Les responsabilités en matière de détention et de renvoi ont été transférées de Citoyenneté et Immigration Canada (CIC) à l'Agence des services frontaliers du Canada au moment de la création de l'ASFC en décembre 2003.

4. Règlement sur l'immigration et la protection des réfugiés (DORS/2002-227). 
Le régime particulier de la détention appliqué aux «étrangers désignés»

Après l'adoption du projet de loi C-31 en juin 2012, la catégorie d'«étranger désigné» a été ajoutée dans la LIPR. Selon les nouvelles dispositions législatives, lorsque le gouvernement désigne l'arrivée au Canada d'un groupe de deux personnes ou plus comme une «arrivée irrégulière», et que, faute de documents en règle, les membres de ce groupe deviennent en conséquence des «étrangers désignés», ces derniers font face à la détention obligatoire (article 55 (3.1) de la LIPR). La détention obligatoire ne s'applique pas aux enfants âgés de 16 ans ou moins, mais les familles seront alors confrontées à un choix difficile: garder la famille unie en détention ou envoyer leurs enfants en famille d'accueil. La désignation d' «arrivée irrégulière» peut être faite avec effet rétroactif au 31 mars 2009 (article 81 (1) du projet de loi C-31). Cela signifie donc que les arrivées de demandeurs d'asile par bateau en octobre 2009 (Ocean Lady) et en août 2010 (Sun Sea) sont couvertes par cette disposition rétroactive.

Le contrôle des motifs de détention des «étrangers désignés» diffère grandement des régimes de contrôle déjà prévus dans la LIPR pour les autres étrangers. Ainsi, la détention des «étrangers désignés» peut être révisée dans les 14 jours uniquement, et ensuite, seulement après 6 mois (art. 57 (1) de la LIPR).

Des garanties constitutionnelles identiques pour tous les étrangers détenus

Depuis l'arrêt Singh ${ }^{5}$ de la Cour suprême du Canada, tout étranger se trouvant sur le territoire canadien est pleinement un sujet de droit et bénéficie de la protection de la Charte canadienne des droits et des libertés (ci-après «La Charte canadienne») ${ }^{6}$. Cela signifie que les garanties juridiques (art. 7 à art. 14 de la Charte), qui sont particulièrement importantes pour la personne en détention, s'appliquent en la circonstance (vie, liberté et sécurité de la personne; protection contre la détention arbitraire; droit d'être informé des motifs de sa détention et droit d'avoir recours sans délai à l'assistance d'un avocat; protection contre tous traitements ou peines cruels et inusités). Aussi, la Charte canadienne exige

5. Singh c. Ministre de l'Emploi et de l'Immigration. (1985). 1 R.C.S. 177.

6. Partie I de la loi constitutionnelle de 1982. 
que les agents de l'ASFC informent toute personne arrêtée ou détenue des motifs de son arrestation ou de sa détention et de son droit à être représentée par un avocat. Enfin, il existe des dispositions spéciales dans la LIPR concernant les mineurs, qui ne devraient être détenus qu'en dernier recours (art. 60 de la LIPR). À cet égard, l'intérêt supérieur de l'enfant (art. 249 du RIPR) doit toujours être pris en compte lors de l'examen de son éventuelle détention (voir sur ce point Nakache, 2011 : 27-29).

En lien avec ce qui précède, les juristes canadiens ont exprimé à plusieurs reprises leurs préoccupations concernant les nouvelles dispositions législatives relatives à la détention des «étrangers désignés» (Association du Barreau canadien, 2010; Association canadienne des avocats et avocates en droit des réfugiés, 2012). En effet, la détention obligatoire est imposée à des personnes qui ne sont pas soupçonnées de criminalité ou de dangerosité. En outre, l'arrivée des demandeurs d'asile peut être désignée d'«irrégulière» simplement parce que le gouvernement soupçonne que les demandeurs d'asile peuvent avoir obtenu leurs documents de voyage de passeurs ou que l'on croit qu'il serait trop long de traiter leur demande selon la procédure normale (Cleveland et al., 2012: 2). Finalement, le régime distinct pour le contrôle des motifs de la détention des «étrangers désignés» est problématique dans la mesure où, comme nous l'avons expliqué ci-dessus, les étrangers détenus bénéficient des mêmes protections constitutionnelles au Canada. Il ne serait donc pas étonnant que les dispositions législatives modifiées par le projet de loi C-31 soient contestées dans un avenir proche devant les tribunaux canadiens (De Grandpré, 2012).

En somme, la catégorie d'«étranger désigné» nouvellement introduite dans la LIPR a pour effet de créer deux régimes de détention distincts pour les demandeurs d'asile. Étant donné que les demandeurs d'asile assujettis à la détention bénéficient des mêmes protections constitutionnelles, il est peu probable que les changements législatifs apportés par C-31 passent le test de constitutionnalité devant les tribunaux.

\section{Le projet de loi C-31: une criminalisation des demandeurs d'asile visant à justifier un recours accru à leur détention}

Il existe aujourd'hui dans les pays d'accueil européens et nordaméricains de plus en plus de «pratiques pénales insidieuses» (Brett, 2005: 31; voir aussi Banks, 2008) visant à «criminaliser» les demandeurs 
d'asile et migrants irréguliers - donc à justifier envers ces derniers des mesures punitives relevant du champ pénal (emprisonnement, surveillance, prise des empreintes digitales, mesures pénales pour la violation d'une loi en matière d'immigration, etc.). La criminalisation des demandeurs d'asile passe nécessairement par leur «délégitimation». En d'autres termes, c'est précisément parce que les demandeurs d'asile sont traités avec suspicion qu'un gouvernement peut adopter des mesures plus sévères à leur égard. Les discours gouvernementaux entourant l'adoption du projet de loi C-31 au Canada illustrent bien ce point: reflétant un processus de «criminalisation» des demandeurs d'asile en cours dans le pays, ils ont permis par le fait même aux autorités gouvernementales d'introduire des politiques de plus en plus répressives envers ce groupe de migrants, notamment à travers la détention.

Peu de temps avant l'adoption du projet de loi C-31, le gouvernement avait multiplié les déclarations publiques visant à faire valoir le bienfondé des nombreuses réformes annoncées (voir sur ce point Showler, 2012). Un discours évocateur en la matière fut celui prononcé par le ministre de l'Immigration lors du dépôt du projet de loi devant la Chambre des communes, en mars 2012. Il s'exprimait alors en ces termes:

Les Canadiens forment un peuple généreux et ouvert d'esprit [...]. C'est la raison pour laquelle les Canadiens s'inquiètent quand ils voient, par exemple, les grandes missions des passeurs de clandestins, comme les deux grands bateaux qui sont arrivés sur la côte ouest du Canada dans les deux dernières années avec des centaines de passagers, des migrants illégaux qui ont payé des réseaux criminels pour être amenés au Canada de façon illégale et très dangereuse [...].

Les Canadiens s'inquiètent également quand ils voient un grand nombre de faux demandeurs d'asile qui n'ont pas besoin de la protection du Canada, mais qui déposent des demandes d'asile parce qu'ils voient dans le système d'asile actuel du Canada une occasion de rester au Canada en permanence et d'avoir accès aux bénéfices sociaux, même s'ils ne sont pas vraiment des réfugiés ayant besoin de la protection de notre pays [...].

Les Canadiens veulent que le Parlement et le gouvernement prennent des mesures énergiques et concrètes pour renforcer l'intégrité et l'équité de nos systèmes d'immigration et de détermination du statut de réfugié, et c'est pour cela que nous avons présenté le projet de loi C-317.

7. Allocution de J. Kenney devant la Chambre des communes, 6 mars 2012 (41 ${ }^{\mathrm{e}}$ législature, $1^{\text {re }}$ session, hansard révisé numéro 090). 
Immédiatement après l'adoption du projet de loi C-31, en juin 2012, le ministre de l'Immigration réitérait:

Cette loi nous aidera à empêcher les criminels étrangers, les passeurs de clandestins et les autres personnes dont la demande d'asile est infondée d'abuser de la générosité du système canadien d'immigration et de bénéficier d'avantages liés à la santé et aux services sociaux financés par les contribuables, a déclaré le ministre Kenney. Le système canadien d'immigration et d'octroi de l'asile est l'un des plus équitables et des plus généreux du monde et cette tradition se poursuivra sous le régime du système amélioré8. (Citoyenneté et Immigration Canada, 2012, para. 2)

Dans ces discours gouvernementaux, une image dominante du demandeur d'asile, qui est d'ailleurs aussi véhiculée par les médias canadiens (Desaulniers, 2012), est celle du «fraudeur» (ou du «faux réfugié»). Cette image permet «la simplification de questions complexes propres à la polémique politique, en désignant des ennemis (les fraudeurs) et en jouant sur le registre d'une indignation morale, disqualifiant ceux qui ne la partageraient pas - forcément "complices" et "laxistes" (Dubois et al., 2003: 14). En d'autres termes, la désignation des demandeurs d'asile en tant que «fraudeurs» permet de mobiliser une "panique morale» (Cohen 1972; voir aussi Welch et Schuster, 2005 ; Banks, 2008) autour des questions compliquées des demandes d'asile et du droit des réfugiés, une panique qui réduit le débat public à un simple clivage entre les «bons» et les «mauvais» réfugiés, dont les derniers seront bien sûr la majorité9.

Un des effets escomptés de la catégorisation des demandeurs d'asile comme «fraudeurs» est leur stigmatisation en tant que personnes «éviantes»:

Social groups create deviance by making the rules whose infraction constitutes deviance, and by applying those rules to particular people and labeling them as outsiders... Deviance is not a quality of the act the person commits but rather a consequence of the application by others of rules and sanctions to an "offender". (Becker, 1963: 9)

8. CIC, «Communiqué - La loi visant à protéger le système d'immigration du Canada reçoit la sanction royale», Ottawa, le 29 juin 2012.

9. Selon la définition consacrée par Cohen (1972), une «panique morale» survient quand un événement ou un groupe de personnes est désigné comme une menace pour les valeurs et les intérêts d'une société. L'une des caractéristiques fondamentale de la «panique morale» est sa capacité à s'autoalimenter, notamment à travers les médias qui amplifient l'importance des enjeux concernés (voir sur ce point Abdelmayek, 1999; Tsoukala, 2002). 
Ainsi, les demandeurs d'asile ne sont plus considérés comme des victimes de persécution, mais plutôt comme une population «dangereuse» pour les sociétés d'accueil, à l'égard de laquelle il devient alors «naturellement» nécessaire de se protéger (Abdelmayek, 1999: 393-413; Banks, 2008; Valluy, 2009). Garland (2001) a bien démontré que la «culture du contrôle» prévaut de plus en plus dans les sociétés d'accueil des pays développés, c'est-à-dire une culture basée sur la gestion des risques et la sécurité des personnes plutôt que sur la réinsertion et les mesures sociales. Il n'est dès lors pas étonnant que les questions migratoires relèvent de plus en plus du champ pénal (Simon, 2007).

Un élément central de la gestion pénale de la migration est sans conteste la détention des demandeurs d'asile:

La détention de ces demandeurs peut être comprise comme une punition «expressive» [c'est-à-dire une punition qui symbolise l'outrance morale des citoyens «moyens» face aux crimes qu' ils sentent comme une menace contre leur sécurité] parce que dans beaucoup de cas [la détention] ne sert pas à un but «utile», mais [sert] plutôt à punir une population étrangère construite comme «menace» à la société nationale. (Freedman, 2007: 3)

Les mesures du projet de loi C-31 ayant trait à la détention illustrent particulièrement bien les propos de Freedman ci-dessus. En effet, comme nous l'avons expliqué précédemment, la LIPR autorise dorénavant la détention automatique des demandeurs d'asile désignés par le ministre de l'Immigration comme faisant partie d'une «arrivée irrégulière» et les assujettit à un régime de contrôle des motifs de détention plus strict. C'est sans compter que la LIPR impose aussi des restrictions particulières aux «étrangers désignés» (échéances plus courtes que les autres demandeurs d'asile pour préparer la revendication du statut de réfugié; interdiction de déposer une demande de résidence permanente pendant 5 ans, même si la personne est reconnue comme réfugiée, etc. $)^{10}$. Injustes, et a priori inconstitutionnelles (voir la partie précédente), les dispositions du projet de loi C-31 ayant trait à la détention des «étrangers désignés» s'avèrent aussi inutiles pour deux raisons principales. Tout d'abord, il a été prouvé que la détention des demandeurs d'asile ne

10. Pour l'analyse de l'ensemble des mesures contenues dans C-31, voir: Conseil canadien pour les réfugiés (2012). Changements au système de détermination du statut de réfugié (C-31). Consulté le 10 novembre 2012, http://ccrweb.ca/fr/la-reforme-refugies. Ce site regroupe l'ensemble des critiques formulées par l'Association canadienne des libertés civiles, l'Association canadienne des avocates et avocats en droit des réfugiés et le Conseil canadien pour les réfugiés concernant C-31. 
dissuade pas ces derniers de recourir au service des passeurs. En effet, du point de vue des demandeurs d'asile, l'effet dissuasif de la détention est bien mince quand les menaces à leur vie et à leur sécurité dans leur pays d'origine sont en jeu (Edwards, 2011 : 1-2). 2). Finalement, il a été démontré que la grande majorité des demandeurs d'asile en détention respectent leurs conditions de mise en liberté (Edwards, 2011). Pour reprendre la formulation de Freedman, les dispositions du projet de loi C-31 sur la détention sont donc une mesure «essentiellement punitive» envers les demandeurs d'asile.

Avec l'adoption de ce projet de loi, l'augmentation du nombre de demandeurs d'asile en détention est inévitable. Dès lors, la question de leurs conditions de détention se pose avec la plus grande acuité. Or, comme nous le démontrons ci-dessous, l'élément de punition d'une supposée «criminalité» des demandeurs d'asile ne se limite pas, au Canada, à l'introduction de mesures visant à accroître leur détention. Il se révèle aussi à travers leur placement en détention dans les prisons provinciales.

\section{Les prisons provinciales: un outil de mise à l'écart et de marginalisation des demandeurs d'asile}

Dans le District de la région du Grand Toronto (Ontario) et dans le Grand Montréal (Québec), les demandeurs d'asile sont détenus dans des centres de surveillance de l'immigration (CSI), destinés exclusivement aux étrangers «à faible risque», et dont la capacité maximale est de 125-150 détenus par CSI ${ }^{11}$. Ailleurs au Canada, dans les endroits où il n'y a pas de CSI, les établissements correctionnels provinciaux et municipaux sont utilisés. En Colombie-Britannique, il existe aussi un CSI à l'aéroport de Vancouver, avec une capacité limitée de 24 lits, mais celui-ci est utilisé uniquement pour les détentions de courte durée: cela signifie qu'après 72 heures, les détenus sont automatiquement transférés dans une prison provinciale de la Colombie-Britannique (Nakache, 2011 : 31).

L'institution carcérale, qui relève du droit pénal, et les centres de détention réservés aux étrangers, qui relèvent du droit administratif,

11. Les détenus «à risque élevé» ou ceux qui présentent des troubles mentaux ou des troubles de comportement, sont habituellement détenus dans des prisons provinciales. Selon l'ASFC, les «personnes jugées à risque élevé sont principalement les personnes qui ont des antécédents criminels, qui sont considérées comme représentant un danger pour le public ou un risque de fuite» ASFC (2012), note 17. 
sont régulièrement abordés dans la littérature scientifique comme deux objets d'étude distincts (Beaulieu-Guérette, 2011: 35). Pourtant, les logiques sous-tendant les deux types d'espace de réclusion convergent en plusieurs points. Au Canada par exemple, sur le plan architectural, les centres de surveillance de l'immigration de l'ASFC sont de plus en plus gérés comme des prisons provinciales (voir sur ce point, dans ce numéro spécial, l'article de Cleveland et al., 2012). Le mode opératoire de la prison est donc progressivement inclus dans celui des centres de surveillance de l'immigration. Inversement, on observe une extension du dispositif des centres de surveillance de l'immigration vers la prison. Plus précisément, comme nous allons l'expliquer dans les lignes qui suivent, les prisons provinciales où sont placés les demandeurs d'asile sont, au même titre que les "camps d'étrangers», un vecteur de mise à l'écart spatial et juridique des demandeurs d'asile. Cette convergence entre le mode de fonctionnement des centres de surveillance de l'immigration de l'ASFC et celui des prisons provinciales remet en question le sens même de la privation de liberté dans chacun des espaces concernés. Par exemple, le centre de surveillance de l'immigration de l'ASFC est censé être un endroit visant uniquement à «immobiliser» les étrangers le temps de statuer sur leur situation administrative ou de les éloigner du territoire, mais la détention dans ces centres prend progressivement les allures d'une peine punitive. Inversement, la prison perd sa fonction de punition ou de réhabilitation lorsqu'elle se substitue à une mesure purement migratoire. Vidée de son sens premier, la «peine» de prison est donc «insensée» pour ceux qui en font l'objet, mais elle devient alors un outil efficace de mise à l'écart social des étrangers «indésirables».

\section{Une mise à l'écart spatiale et juridique}

Afin de bien comprendre les logiques communes qui sous-tendent les différents espaces de privation de liberté spécifique aux étrangers, le concept de «camp d'étrangers» (Agamben, 1997, 2003; Le Cour Grandmaison et al., 2007; Agier, 2008; Bernardot, 2008) s'avère utile. Les caractéristiques propres aux camps d'étrangers pourraient être définies de la sorte:

Espaces de réclusion pour étrangers enfermés sans jugement, hors du système pénitentiaire, dû à leur simple statut administratif d'étranger [...]. 
La fonction des camps varie, mais il s'agit généralement d'isoler, de faire un tri, de ficher, de bloquer, d'expulser ou parfois simplement de réorienter les trajectoires des migrants indésirables. (Beaulieu-Guerette, 2011:29)

Parmi les types de camps d'étrangers au Canada, on retrouve les centres de surveillance de l'immigration, qui sont administrés par l'ASFC. En mars 2012, des agents d'immigration de l'ASFC annonçaient que le CSI de Toronto est en rénovation et qu'il y aurait 80 à 100 lits supplémentaires dans un avenir proche. Ils indiquaient qu'ils anticipent «des déportations massives ${ }^{12} »$ qui commenceront sous peu du fait des modifications récentes à la LIPR (Godfrey, 2012). Les centres de surveillance de l'immigration sont donc utilisés comme un instrument de mise à distance des étrangers.

L'institution carcérale est également un outil de mise à distance des étrangers, et ce, pour deux raisons principales:

- Tout d'abord, même si la majorité des «réfugiés $»^{13}$ sont détenus en moyenne pour une période n'excédant pas 10 jours (Nakache, 2011 : $44)$, des statistiques plus précises révèlent que les «réfugiés» dans les prisons provinciales restent en détention plus longtemps que les «réfugiés» détenus dans les centres de surveillance de l'immigration. Par exemple, $36 \%$ des «réfugiés» dans les prisons provinciales étaient en détention pour plus de 40 jours; par comparaison, la proportion était de $12 \%$ pour les «réfugiés» détenus dans les centres de surveillance de l'immigration de l'ASFC (Nakache, 2011 : 45-46). Évidemment, ces statistiques constituent des moyennes, et il arrive parfois que les «réfugiés» soient détenus dans les centres de l'ASFC pour de très longues périodes. Toutefois, étant donné que le gouvernement n'explique pas les causes de cette disparité, ces chiffres pourraient laisser penser, comme cela a été avancé ailleurs (Badinter, 2004: 21), que le placement des étrangers en établissement pénitentiaire plutôt que dans les centres de détention pour étrangers permettrait aux

12. Traduction libre de: «mass deportations».

13. Il importe ici de préciser que les seules données gouvernementales disponibles concernant la détention des demandeurs d'asile au Canada, et que l'on retrouve dans les statistiques de l'ASFC sous la dénomination «réfugiés», regroupent deux catégories distinctes: les revendicateurs du statut de réfugié (communément appelés «demandeurs d'asile») et ceux dont la demande d'asile a été refusée et qui sont en attente de renvoi. Il est donc impossible, à ce jour, d'obtenir des statistiques précises concernant uniquement la détention des «demandeurs d'asile» au Canada, et c'est la raison pour laquelle le terme «réfugiés» est utilisé dans cet article. 
autorités de disposer de plus de temps pour mettre en œuvre leurs procédures d'immigration, notamment l'éloignement du territoire.

- Ensuite, l'utilisation des prisons a augmenté au cours des dernières années. Entre 2005 et 2009, une moyenne de $23 \%$ des «réfugiés» étaient détenus dans des prisons provinciales, alors qu'en 2009-2010, ils étaient 29 \% (ASFC, 2010a; Nakache, 2011 : 47-48). Étant donné que moins de $6 \%$ des «réfugiés» étaient détenus pour des raisons de criminalité, de risque de sécurité ou de danger pour le public (Nakache, $2011: 47-48)$, cela signifie que la très vaste majorité des «réfugiés» détenus dans les prisons à cette période étaient des prisonniers à «faible risque». Hormis l'expansion ponctuelle du centre de surveillance de l'immigration de Toronto, l'ASFC ne prévoit pas augmenter «dans l'immédiat» sa capacité de détention des centres de surveillance de l'Immigration, du fait de «l'accroissement important des ressources que cela exige[rait]» (ASFC, 2009, réponse à la recommandation 4). L’ASFC prévoit toutefois «résoudre la question dans les limites des ressources actuelles», en «se tourn[ant] vers des partenaires provinciaux pour répondre à ses besoins en matière de détention» (ASFC, 2009). Les centres correctionnels continueront donc, dans un futur proche, à être utilisés pour détenir des demandeurs d'asile, et ce, malgré que les provinces aient annoncé à plusieurs reprises qu'elles prévoient un débordement de leurs institutions carcérales suite à l'entrée en vigueur du projet de loi C-10 sur la réforme des peines ${ }^{14}$.

En somme, les prisons provinciales constituent, au même titre que les centres de surveillance de l'immigration de l'ASFC, un des principaux outils de mise à distance des étrangers indésirables. Certains auteurs (Agamben, 1997, 2003; Bernardot, 2008) considèrent en outre que les «camps d'étrangers» placent les migrants dans un état juridique d'«exception ordinaire», et donc dans une situation de «non-droit» quasi permanente. A priori, puisque les prisons s'insèrent dans un système de justice très institutionnalisé, et qu'elles sont régies par des lois strictes et codifiées, elles ne correspondent pas à une zone de «nondroit». Pourtant, l'expérience des demandeurs d'asile détenus dans les prisons est, à plusieurs égards, marquée par l'exception, puisque les

14. Projet de loi C-10, Loi sur la sécurité des rues et des communautés, première session, quarante et unième législature, 60 Elizabeth II, 2011 (sanction royale le 12 mars 2012). 
conséquences de leur séjour en détention sont bien souvent arbitraires. Pour illustrer ce point, nous allons nous pencher sur la situation en Colombie-Britannique. Avec les nouvelles dispositions législatives ayant trait à la détention des "étrangers désignés», il risque d'y avoir une augmentation des détentions en Colombie-Britannique, puisque la province est la destination la plus probable pour les arrivées en bateau. En Colombie-Britannique, tous les demandeurs d'asile sont - sans exception - détenus dans des institutions carcérales, le plus souvent avec les prisonniers de droit commun. Il a été démontré ailleurs (Nakache, 2011) que cette situation ne respecte pas les dispositions du droit international, car les demandeurs d'asile doivent être détenus dans des conditions conformes à leur statut, et "pas comme des personnes présumées ou reconnues coupables de délits (à moins qu'ils ne soient eux-mêmes présumés ou reconnus coupables)» (Haut Commissariat des Nations Unies pour les réfugiés, 1999, para. 26). Toutefois, notre objectif ici est de mettre l'accent sur le flou entourant la frontière entre droit pénal et droit administratif en ce qui concerne les demandeurs d'asile dans les prisons, qui a pour effet de les mettre juridiquement à l'écart.

Après trois jours passés en détention au CSI de Vancouver, les demandeurs d'asile sont automatiquement transférés dans une prison provinciale de la Colombie-Britannique. Bien qu'il existe des prisons à sécurité moyenne dans la province, tous les demandeurs d'asile sont placés, sans raison valide, dans des prisons à sécurité maximale. Ils sont soumis, au même titre que n'importe quel autre prisonnier de droit commun, à l'ensemble des règles institutionnelles. Ainsi, contrairement aux demandeurs d'asile détenus dans les centres de l'ASFC, ils doivent porter l'uniforme de prisonnier, et leur liberté de mouvement est très restreinte (Nakache, 2011: 76). En outre, les services correctionnels n'accordent aucun traitement de faveur aux demandeurs d'asile - les gardiens de prison n'ont d'ailleurs aucun moyen d'identifier qui, parmi les migrants détenus, est un demandeur d'asile. Les besoins spécifiques des demandeurs d'asile ne sont donc pas pris en considération. Par exemple, contrairement aux demandeurs d'asile détenus dans les centres de l'ASFC, les appels téléphoniques des demandeurs d'asile emprisonnés font l'objet de nombreuses restrictions: ils peuvent avoir lieu uniquement lorsque ces derniers sont dans la salle commune (donc à des moments très précis de la journée), et étant donné que les appels sont sur écoute, ils peuvent être arrêtés à n'importe quel moment. De plus, les appels locaux sont gratuits pour les demandeurs d'asile détenus aux 
CSI, mais ils sont payants pour ceux détenus en institution carcérale. Quant aux appels internationaux, ils ne sont possibles qu'avec une carte d'appel délivrée par l'institution pénitentiaire, mais les cartes d'appel ne fonctionnent pas toujours pour téléphoner dans les pays d'origine des demandeurs d'asile (Nakache, 2011 : 76-77). Dans ces conditions, il est difficile pour les demandeurs d'asile de réunir les documents nécessaires à leur revendication du statut de réfugié, d'autant plus qu'ils peuvent rarement bénéficier d'une aide extérieure: aucune ONG n'a l'autorisation de rendre visite aux demandeurs d'asile en prison, et il est n'est pas facile pour ces derniers d'obtenir les services d'un avocat (Nakache, 2011: 67-72).

La situation de grande vulnérabilité des demandeurs d'asile dans les prisons provinciales est exacerbée par le «flou juridique» entourant le partage du champ des compétences entre le fédéral et le provincial. Par la loi sur l'immigration, l'ASFC est l'autorité fédérale habilitée à détenir les demandeurs d'asile. Par la Loi constitutionnelle de $1867^{15}$, la province est toutefois responsable des soins, de la garde et du contrôle des demandeurs d'asile détenus dans les prisons provinciales. Cela signifie que même si l'ASFC détient le pouvoir de décision concernant la détention d'un demandeur d'asile, elle n'a pas de contrôle sur la manière dont les services correctionnels provinciaux gèrent leurs institutions carcérales (Nakache, 2011 : 87-93). L'utilisation des dispositifs de contention pour le transport des détenus illustre bien l'impact négatif de ce flou juridique sur les demandeurs d'asile: alors que l'ASFC a pour politique de ne pas utiliser les dispositifs de contention avec les femmes enceintes, l'ASFC n'a toutefois pas encore été en mesure de dissuader les services correctionnels de la Colombie-Britannique de ne pas menotter ni enchaîner les demandeuses d'asile enceintes (Nakache, 2011 : 89). Les demandeurs d'asile sont donc mis à l'écart par le fonctionnement même $\mathrm{du}$ droit canadien : ils constituent l'exception au principe de séparation du champ des compétences, et dans leur cas, la frontière entre droit administratif et droit pénal est trouble.

En conclusion, les prisons provinciales constituent, au même titre que les «camps d'étrangers», un instrument de mise à l'écart des demandeurs d'asile indésirables et un moyen d'appliquer envers eux un régime d'exception. Le sens premier de la peine carcérale est donc progressivement détourné, pour se rapprocher de celui propre aux camps.

15. Loi constitutionnelle de $1867,30 \& 31$ Victoria, c 3, articles 91 et 92. 


\section{Une ségrégation sociale}

Il existe grosso modo deux traditions de philosophie pénale (Foucault, 1975; Combessie, 2004; Lalande, 2006). La première considère que le propre de l'institution carcérale est d'agir comme sanction punitive. En ce sens, la prison servirait uniquement à expier une peine: «Le fait que l'on impose une punition à une personne se fonde sur l'idée pure du châtiment. Le contrevenant doit expier sa faute afin de rétablir un équilibre moral. La sanction n'a donc aucune valeur utilitaire. On punit parce que c'est une obligation morale de punir et on punit strictement en vertu de ce qui s'est passé antérieurement. C'est la punition pure» (Lalande, 2006: 33). La seconde philosophie considère que la prison doit se révéler «utile» pour l'individu et la société, et qu'elle doit donc favoriser la réduction des comportements prohibés. La prison aurait alors trois fonctions: 1) la dissuasion générale (infliger une peine pour dénoncer un comportement répréhensible et envoyer un message à la société) et/ou la dissuasion individuelle (prévenir la récidive de l'individu) ; 2) la neutralisation (emprisonner le contrevenant dans un milieu sécuritaire afin qu'il ne puisse plus menacer la population); et 3) la réhabilitation (la sanction est établie en fonction de l'individu - et non en fonction du délit commis - et elle vise à modifier le comportement).

Quelle que soit notre conception des différentes fonctions traditionnelles de la prison, et de la manière dont ces fonctions auraient évolué dans le temps (voir sur ce point Garland, 2001), un simple constat s'impose: étant donné que la prison, qui relève du droit pénal, est une sentence destinée à l'individu qui a commis un crime, la détention des demandeurs d'asile dans les institutions carcérales pour des raisons purement administratives ne permet pas à la prison de remplir une de ses trois fonctions traditionnelles.

Détournée de sa fonction première, quel est alors le but recherché de la détention lorsquappliquée aux demandeurs d'asile, par opposition au reste des détenus? Certes, la prison invalide toute prétention de réhabilitation et de réinsertion, mais elle exerce alors une fonction sociale ségrégative importante envers les demandeurs d'asile. Wacquant (1999: 64) explique:

Cette situation traduit la plus grande sévérité de l'institution pénale à leur égard. Il s'agit bien là en priorité d'un emprisonnement de différenciation ou de ségrégation, visant à maintenir un groupe à l'écart et à faciliter sa 
soustraction du corps sociétal..., par opposition à l'«enfermement d'autorité » et l'«enfermement de sûreté».

La prison contribue donc à la marginalisation des demandeurs d'asile, «indésirables parmi les indésirables» (Beaulieu-Guérette, 2010: 103), en produisant simultanément deux images stéréotypées: celle de l'étranger qui est vu comme un délinquant en puissance (Abdelmalek, 1999); et celle du criminel qui est considéré comme «étranger» à la société (Garland, 2001). Dès lors, «la frontière sociale de la prison et la frontière géographique des camps se confondent : à travers ces deux dispositifs de relégation spatiale, on rappelle aux étrangers enfermés que leur place est [à la fois] en marge de la société et du territoire national» (Beaulieu-Guérette, 2010: 109).

En conclusion, malgré les nombreuses différences apparentes entre le «camp d'étrangers» et la prison, il semble aujourd'hui pertinent de mettre en parallèle ces deux dispositifs de mise à l'écart spatiale, juridique et sociale afin de voir qu'ils sont reliés en de nombreux points.

\section{Conclusion}

Au Canada, la question de l'asile est devenue récemment un sujet de controverse et de polémique, et ce sont les autorités publiques ellesmêmes qui ont contribué à la fabrication d'une population "problématique» de demandeurs d'asile. La «criminalisation» des demandeurs d'asile a permis aux instances publiques canadiennes d'introduire des politiques punitives à leur égard. L'une d'entre elles est la détention, qui constitue une stratégie de gestion de ces populations. Les études sur la prison et celles sur les «camps d'étrangers» sont prolifiques, mais elles se croisent très rarement. Dans cet article, nous avons décidé de mettre l'accent sur une situation méconnue - la détention des demandeurs d'asile en institution carcérale au Canada - afin d'offrir une réflexion sur les logiques convergentes entre les deux formes distinctes d'enfermement des étrangers que sont la prison et le camp d'étrangers. Nous avons pu constater que la prison exerce, au même titre que les camps d'étrangers, une fonction de ségrégation spatiale et juridique. Nous avons aussi démontré qu'elle permet de marginaliser les demandeurs d'asile au sein de l'espace social. S'éloignant de ses fonctions traditionnelles, la prison contribue donc à marquer les demandeurs d'asile comme «indésirables». 
Les effets pervers de ce dispositif sur les demandeurs d'asile sont nombreux. L'un d'entre eux est l'érosion de leurs droits fondamentaux. Dans un contexte marqué par une volonté gouvernementale de recourir de manière accrue à la détention, un examen étroit des tribunaux s'avère donc plus que jamais nécessaire.

\section{Références}

Abdelmalek, S. (1999). Immigration et «pensée d'État». In S. Abdelmalek (ed.), La double absence: des illusions de l'émigré aux souffrances de l'immigré(395-413). Paris: Seuil.

Agamben G. (1997). Homo Sacer I. Le pouvoir souverain et la vie nue. Paris: Seuil. Agamben G. (2003). État d'exception. Homo Sacer II. Paris: Seuil.

Agence des services frontaliers du Canada. (2009). Réponse du gouvernement au rapport du Comité permanent des comptes publics. Le chapitre 7, les détentions et les renvois - Agence des services frontaliers du Canada du Rapport de mai 2008 de la vérificatrice générale du Canada (Rapport n9). Ottawa, ON: Parlement du Canada.

Agence des services frontaliers du Canada. (2010a). Detentions at a Glance, 20052010. Ottawa: Canada.

Agence des services frontaliers du Canada. (2010b). Programmes de détention et de renvoi de l'ASFC - Étude d'évaluation (Rapport final). Consulté le 23 janvier 2013, http://www.cbsa-asfc.gc.ca/agency-agence/reports-rapports/ ae-ve/2010/dr-rd-fra.html.

Agier, M. (2008). Gérer les indésirables. Des camps de réfugiés au gouvernement humanitaire. Paris: Éditions Flammarion.

Association canadienne des avocats et avocates en droit des réfugiés. (2012). Projet de loi C-31: Le ministre dit une chose, son projet de loi en dit une autre. Consulté le 23 janvier 2013, http://www.refugeelawyersgroup.ca/ theministersays\#FR.

Association du Barreau canadien. (2010). Projet de loi C-49, Loi visant à empêcher les passeurs d'utiliser abusivement le système d'immigration canadien. Consulté le 23 février 2012, http://www.cba.org/ABC/Memoires/pdf/10-78-fr.pdf.

Badinter, R. (2004). Étude sur les étrangers détenus. Paris: Commission nationale consultative des droits de l'Homme (CNCDH).

Banks, J. (2008). The Criminalisation of Asylum Seekers and Asylum Policy. Prison Service Journal, 175, 43-49.

Bauman, Z. (1991). Modernity and Ambivalence. Oxford: Polity Press.

Beaulieu-Guérette, E. (2010). Étrangers derrière les barreaux: la prison dans le dispositif de mise à l'écart des étrangers indésirables en France. Consulté le 23 mars 2012, http://www.reseau-terra.eu/article1062.html.

Becker, H. (1963). Outsiders: Studies in the sociology of deviance. New York: Free Press. 
Bernardot, M. (2008). Camps d'étrangers. Bellecombe-en-Bauges: Éditions du Croquant.

Bigo, D. (1998a). L'immigration à la croisée des chemins sécuritaires. Revue européenne des migrations internationales, 14 (1), 25-46.

Bigo, D. (1998b). Sécurité et immigration: vers une gouvernementalité par l'inquiétude? Cultures et Conflits, 31/32, 39-62.

Blandy, S., Lister, D., Atkinson, R. \& Flint, J. (2003). Gated communities: A systematic review of the research evidence (Summary). Swindon: ESRC Centre for Neighbourhood Research.

Brett, S. (2005). Politics as usual: The criminalization of asylum seekers in the United States. RSC working paper ${ }^{\circ} 26$. Consulté le 23 janvier 2013, http://www.rsc.ox.ac.uk/publications/working-papers-folder_contents/ RSCworkingpaper26.pdf.

Cheng, S. (2004). Law, justice, and power: Between reason and will. Stanford: Stanford University Press.

Citoyenneté et Immigration Canada. (2012). Communiqué - La Loi visant à protéger le système d'immigration du Canada reçoit la sanction royale. Consulté le 23 janvier 2013, http://www.cic.gc.ca/francais/ministere/media/communiques/ 2012/2012-06-29.asp.

Cleveland J., Rousseau C. \& Kronick R. (2012). L'impact du projet de loi C-4 sur la santé mentale des demandeurs d'asile. Consulté le 10 mars 2012, http://www. csssdelamontagne.qc.ca/fileadmin/csss_dlm/Publications/Publications_ CRF/Impact_du_projet_de_loi_C-4_sur_la_sante_mentale_des_demandeurs _d_asile_integral.pdf

Cohen, S. (1972). Folk Devils and Moral Panics. London: MacGibbon and Kee.

Combessie, P. (2004). Sociologie de la prison, Paris: La Découverte.

Courau, H. (2012). Altérisation et mise en camp des surnuméraires: l'expérience de Sangatte. In C. Crenn \& L. Kotobi (eds.), Du point de vue de l'ethnicité. Armand Collin: Paris.

Crépeau, F. \& Nakache, D. (2006). Controling irregular migration in Canada: Reconciling security concerns with human rights protection. IRPP Choices, 12(1).

De Grandpré, H. (2012, mars 26). Demandes d'asile: le projet de loi C-31 sera contesté devant les tribunaux. La Presse. Consulté le 23 janvier 2013, http:// www.lapresse.ca/actualites/quebec-canada/politique-canadienne/201203/ 26/01-4509457-demandes-dasile-le-projet-de-loi-c-31-sera-contestedevant-les-tribunaux.php

Derrida, J. (1968). La Différance. Théorie d'ensemble. Paris: Seuil.

Derrida, J. (1992). The Other Heading : Reflections on Today's Europe. Bloomington: Indiana University Press.

Desaulniers, M. (2012). Refugee as sovereign value: Staunching the flow in a time of drought (Unpublished thesis in Master of Arts). Simon Fraser University.

Dubois, V., Buton, F., Chambolle, L. \& Dulong, D. (2003). Les conditions sociales de la rigueur juridique (Dossiers d'études $n^{\circ} 48$ ). Paris: Caisse nationale d'allocations familiales. 
Edwards, A. J. (2011). Back to basics: The right to liberty and security of person and "alternatives to detention" of refugees, asylum-seekers, stateless persons and other migrants (PPLA/2011/01.Rev.1). Genève: UN High Commissioner for Refugees.

Faist, T. (2002). "Extension du domaine de la lutte": International Migration and Security before and after September 11, 2001. International Migration Review, 36 (1), 7-14.

Fischer, N. \& Darley, M. (2010). Le traitement de l'immigration, entre logique administrative et logique pénale. Champs pénal, 7, 1-15.

Foucault, M. (1975). Surveiller et punir. Naissance de la prison. Paris: Gallimard.

Freedman, J. (2007, juin). La criminalisation des demandeurs d'asile: une étude comparative des politiques de détention/rétention en France et en Grande-Bretagne. Acte du colloque international «La fabrique de populations problématiques par les politiques publiques», Nantes.

Garland, D. (2001). The culture of control: Crime and social order in contemporary society. Chicago: University of Chicago Press.

Global Detention Project. (2012). Immigration Detention in Canada. A Global Detention Project Special Report. Genève: Global Detention Project.

Godfrey T. (2012, mars 17). Bogus refugee detention space boosted. Toronto Sun. Consulté le 23 janvier 2013, http://www.torontosun.com/2012/03/17/ bogus-refugee-detention-space-boosted.

Haut Commissariat des Nations Unies pour les réfugiés. (1999). Détention des demandeurs d'asile et des réfugiés: le cadre, le problème et la pratique recommandée (Publication $\mathrm{n}^{\circ}$ EC/49/SC/CRP.13.). Consulté le 10 mars 2012, http:// www.unhcr.org/refworld/docid/4ae9ac8fd.html

Huysmans, J. (2006). The Politics of Insecurity: Fear, Migration and Asylum in the $E U$. London: Routledge.

Kapur, R. (2007). The citizen and the migrant: Postcolonial anxieties, law, and the politics of exclusion/inclusion. Theoretical Inquiries in Law, 8 (2), 537570.

Lalande, P. (2006). Punir ou réhabiliter les contrevenants: du «Nothing Works» au «What Works» (Montée, déclin et retour de l'idéal de réhabilitation). In P. Lalande \& O. Lamalice (eds), La sévérité à l'beure du populisme (36-77). Québec: Ministère de la Sécurité publique.

Le Cour Grandmaison, O., Lhuilier G. \& Valluy, J. (2007). Le retour des camps? Sangatte, Lampedusa, Guantanamo. Paris: Éditions Autrement.

Nakache, D. (2011). The human and financial cost of detention of asylumseekers in Canada. Study researched and written for UNHCR. Consulté le 23 février 2012, http://www.socialsciences.uottawa.ca/edim/eng/documents/ DNakache-FINALREPORT-December2011.pdf

Scherrer, A., Guittet, E-P. \& D. Bigo. (2010) Mobilités sous Surveillance. Perspectives croisées UE-Canada. Montréal: Athéna.

Sholwer, P. (2012). Bill C-31: A big new refugee bill but the same old debate. Consulté le 02 mars 2012, http://maytree.com/blog/2012/02/bill-c-31-a-big-newrefugee-bill-but-the-same-old-debate/ 
Simon, J. 2007. Governing through crime: How the war on crime transformed american democracy and created a culture of fear. Oxford: Oxford University Press.

Soguk, N. (1999). States and Strangers: Refugees and Displacements of Statecraft. Minneapolis: University of Minnesota Press.

Tsoukala, A. (2002). Le traitement médiatique de la criminalité étrangère en Europe. Déviance et Société, 26, 61-82.

Tsoukala, A. \& Ceyhan, A. (2001). Contrôle de l'immigration: mythes et réalités. Cultures $\mathfrak{F}$ Conflits, 26-27. Consulté le 24 février 2012, http:// conflits.revues.org/index356.html.

Valluy, J. (2009). Rejet des exilés - Le grand retournement du droit de l'asile. Paris: Éditions du Croquant.

Wacquant, L. (1999). Des ennemis commodes. Persée, 129 (1), 63-67.

Welch, M. \& Schuster, L. (2005). Detention of asylum seekers in the UK and USA: Deciphering noisy and quiet constructions. Punishment Society, 7(4), 397-417.

\section{Case Law}

Singh c. Ministre de l'Emploi et de l'Immigration. (1985). 1 R.C.S. 177

\section{Les lois et règlements}

Loi constitutionnelle de 1867. $30 \& 31$ Victoria, ch. 3 (R.U.).

Loi sur l'immigration et la protection des réfugiés. L. C. 2001, ch. 27.

Loi visant à protéger le système d'immigration du Canada. L.C. 2012, ch. 17.

Règlement sur l'immigration et la protection des réfugiés (DORS/2002-227). Consulté le 23 janvier 2013, http://laws-lois.justice.gc.ca/fra/reglements/DORS2002-227/TexteComplet.htm1

ABSTRACT - This paper examines the detention of asylum seekers in Canada and the ways in which penal and administrative mechanisms are increasingly used together in the area of immigration. We show that the "criminalization" of asylum seekers facilitates the implementation of punitive measures against asylum seekers, such as increased use of detention. A strong focus of this paper is on the use of provincial prisons to confine asylum seekers in administrative detention (one third of asylum seekers with a non-criminal status are held in penal institutions). As we show, criminal facilities carry out the same functions as camps: they contribute to the spatial, legal and social segregation of asylum seekers, marking them therefore as "unwanted" migrants.

KEYWORDS - Detention, Canada, asylum seekers, human rights, criminalization of immigration.

RESUMEN - El objetivo del presente artículo, que trata sobre la detención de los demandantes de asilo en Canadá, es de poner de relieve las lógicas convergentes entre el tratamiento penal y administrativo de la inmigración. Demostramos que la criminalización actual de los demandantes de asilo facilita la implementación de políticas 
públicas cada vez más represivas hacia éstos, precisamente a través de un creciente recurso a la detención. Nos interesamos también en la encarcelación de los demandantes de asilo en prisiones provinciales, mientras que el motivo de dicha detención es puramente "administrativo» (es decir que los demandantes de asilo no son presuntos autores de delitos, ni han sido reconocidos como culpables de éstos): este fenómeno no es menor, ya que implica un demandante de asilo sobre tres en Canadá. Mostramos, entonces, que la institución carcelaria ejerce, al mismo titulo que los «campos para extranjeros», una función de segregación espacial, jurídica y social que tiene por objetivo el señalar los demandantes de asilo como una población de personas indeseables.

PALABRAS CLAVE - Detención, Canadá, demandantes de asilo, derechos humanos, criminalización de la inmigración. 